(e-migrinter)

e-Migrinter

12 | 2014

Les chiffres de l'immigration : un regard critique sur les statistiques

\title{
Les nouveaux migrants au Liban : vers une ethnicisation du marché de l'emploi
}

Thèse soutenue le 20 décembre 2012 à l'Université de Poitiers, en cotutelle avec l'Université Libanaise

Suzanne Menhem

\section{OpenEdition}

Journals

Édition électronique

URL : https://journals.openedition.org/e-migrinter/378

DOI : $10.4000 /$ e-migrinter.378

ISSN : 1961-9685

Éditeur

UMR 7301 - Migrinter

Édition imprimée

Date de publication : 15 octobre 2014

Pagination : 86-90

ISSN : 1961-9685

Référence électronique

Suzanne Menhem, "Les nouveaux migrants au Liban : vers une ethnicisation du marché de l'emploi », e-Migrinter [En ligne], 12 | 2014, mis en ligne le, consulté le 20 mai 2021. URL : http://

journals.openedition.org/e-migrinter/378 ; DOI : https://doi.org/10.4000/e-migrinter.378 


\title{
Les nouveaux migrants au Liban : vers une ethnicisation du marché de l'emploi
}

\author{
Thèse soutenue par Suzanne Menhem le 20 décembre 2012 à l'Université de \\ Poitiers, en cotutelle avec l'Université Libanaise
}

C ette thèse s'intéresse à la présence des migrants arrivés entre 1990 et 2012 au Liban à partir de quatre groupes de nationalités différentes: les Sri-Lankais(es), les Éthiopien(ne)s, les Philippin(e)s et les Indien(ne)s. Elle vise à mettre en lumière les formes et les implications de la place croissante que ces individus occupent sur le marché de l'emploi libanais, caractérisée par un phénomène d'ethnicisation de certains secteurs d'activité. Il s'agit également d'évaluer l'impact de la présence de cette main-d'œuvre étrangère sur une population au sein de laquelle existent des tensions ethniques et religieuses et sur les transformations que ces nouveaux migrants entraînent dans la société libanaise - en particulier sur le marché de l'emploi - ainsi que de reconstituer les conditions de vie et les pratiques culturelles et cultuelles de ces étrangers.

Le système migratoire libanais est caractérisé par un double mouvement d'émigration et d'immigration. Dans ce contexte nous nous sommes tout d'abord intéressée :

- À la place et à la spécialisation ethnique des migrants africains et asiatiques sur le marché du travail et dans la société libanaise.

- Puis, dans une perspective comparatiste, une présentation des conditions de vie de ces différents migrants a permis de mieux saisir les changements que leur présence instaure dans la société libanaise.
Dans ce travail de recherche, il s'agissait de s'appuyer sur les travaux issus de la sociologie des circulations migratoires et des mobilités humaines (Domenach, Picouet, 1987 ; Courgeau, 1988 ; Ma Mung, Doraï, Hily, 1998 ; Sassen, 1999 ; Dureau, 2002) pour analyser la situation de cette main d'œuvre étrangère devenue importante sur le marché du travail libanais. Cette migration se caractérise par la présence de travailleurs temporaires dont certains ont des titres de séjour en règles et d'autres sont en situation « irrégulière ».

La notion d'ethnicisation a été mobilisée en tant qu'outil conceptuel permettant la description et l'analyse des modes de présence des populations migrantes sur le marché de l'emploi à partir d'une démarche prenant en compte différentes échelles. En effet, le Liban est devenu un pays importateur de maind'œuvre dans l'espace migratoire moyenoriental. Pour expliquer ce phénomène, il faut d'abord comprendre la place de ces nouveaux migrants sur le marché de l'emploi libanais, en s'interrogeant tout particulièrement sur l'ethnicisation de certains métiers. Nous explorons aussi la diversité des enjeux des pratiques culturelles sur l'intégration des migrants, les stratégies et les politiques d'emploi du gouvernement et des employeurs, ainsi que les changements sociaux à l'œuvre dans une société déjà profondément marquée par des divisions. Les mutations qui se sont produites et qui se poursuivent aujourd'hui nous paraissent être un phénomène important à saisir dans le domaine des migrations internationales. 
Notre recherche prend pour point de départ les questions suivantes: dans quelle mesure l'arrivée des nouveaux migrants sur le marché de l'emploi traduit-elle, de nos jours, différentes formes de recomposition de la société libanaise ? Dans quelle mesure assistons-nous à des processus d'ethnicisation de certains métiers occupés par ces nouvelles populations immigrées? Comment expliquer qu'un pays d'émigration comme le Liban connaisse un taux aussi élevé d'immigration?

Pour répondre à ces questions, nous avons jugé pertinent de faire appel à une démarche méthodologique qui s'inscrit dans la sociologie et l'ethnographie, autorisant ainsi une démarche essentiellement qualitative.

Nos enquêtes ont révélé l'hétérogénéité des provenances et la pluralité des activités des migrants. L'échantillon de notre enquête est composé de 152 migrants et de 70 responsables institutionnels, administratifs (ministère du Travail, ministère de l'Intérieur, Sûreté Générale et ambassades indienne, srilankaise, philippine et éthiopienne à Beyrouth), associatifs et caritatifs (Caritas migrants, Centre afro-asiatique, institutions religieuses). Les enquêtes ont été menées dans plusieurs lieux de vie et de travail de ces immigrés tels que les églises, les espaces festifs, les marchés, les boutiques de produits alimentaires, les magasins de musique ou les salons de coiffure. Elles se sont principalement déroulées le week-end, notamment les dimanches, jours de prières ou de messes, de fêtes et de rassemblement divers. Nous avons observé les immigrés dans leur vie quotidienne. Enfin, nous avons constaté l'appropriation, par la population migrante de nouveaux espaces collectifs telles les zones de commerces.

Notre travail de bibliographie - au-delà de procéder à une connaissance fine de la situation migratoire au Liban - visait à dresser une typologie des emplois occupés par les immigrés liés à notre étude. Ainsi, nous nous sommes efforcés de décrire et d'analyser au mieux l'intégration des migrants interrogés.

Dans la première partie de la thèse et après avoir rappelé que l'émigration revêt un caractère particulier depuis le XIX ${ }^{\text {ème }}$ siècle de par son importance numérique par rapport à la population libanaise, nous soulignons qu'au cours des deux dernières décennies le Liban a été marqué par une immigration relativement importante, mais aussi par des migrations de transit. Ce double processus remonte au début des années 1970, lorsque le pays a commencé à accueillir une main d'œuvre-étrangère à la fois arabe et non arabe. La présence accrue des travailleurs étrangers au Liban s'est déroulée en différentes vagues migratoires alors que, dans le même temps, les changements socio-économiques ont affecté en profondeur l'immigration vers le Liban. En outre depuis la fin de la guerre civile, le pays a eu recours à la main d'œuvre asiatique. Les besoins en travailleurs étant considérables en raison des destructions matérielles liées au conflit qui s'est déroulé entre 1975 et 1991 et de l'importante émigration libanaise qui s'est opérée au cours de cette même période, les migrations asiatiques vers le Liban ont pris différentes formes (migrations de travail, temporaires et régulières).

Dans la seconde partie, nous nous intéressons aux conséquences de l'immigration sur le marché du travail libanais. Nous mettons l'accent sur le rapprochement entre l'impact du phénomène migratoire sur les modes d'organisation du marché de l'emploi et la concentration des travailleurs immigrés dans certaines branches et l'évolution des activités professionnelles des immigrés. Cette partie inclut par ailleurs une série d'analyses concernant les mesures relatives au statut des travailleurs étrangers au Liban. Plus particulièrement, le premier chapitre analyse les caractéristiques du marché de l'emploi au 
Liban qui s'avère, prédisposé à l'ethnicisation de la distribution des emplois. En effet, le marché de l'emploi libanais se caractérise par une pénurie de main d'œuvre dans les métiers non qualifiés que des étrangers viennent pallier. Sur le marché de l'emploi des immigrés, l'origine détermine bien souvent le métier que ces derniers exercent. Tandis que les Sri-Lankais, les Népalais, les Philippins, les Éthiopiens, les Bangladais réalisent souvent des travaux domestiques, les Syriens, les Égyptiens et les Indiens travaillent fréquemment dans la construction, dans l'agriculture ou dans l'industrie. Nous constatons donc dans certains métiers une concentration de certaines nationalités que l'embauche de nouveaux migrants par des ressortissants de même origine renforce et qui apparait comme un phénomène très important au Liban. En 2011, selon les statistiques officielles du ministère du Travail, il y a 34653 Éthiopiens, 29049 Philippins, 24000 Bangladais, 12527 Sri Lankais, 11579 Népalais, 3470 Malgaches et 5283 ressortissants d'autres pays ${ }^{1}$. Par ailleurs, les femmes sont majoritaires dans les services domestiques et d'aide à la personne, alors que les hommes se retrouvent plutôt dans le bâtiment, dans le gardiennage, et dans d'autres activités considérées comme masculines. D'autre part, les emplois des travailleurs étrangers sont souvent caractérisés par des conditions de travail extrêmement difficiles : nombre d'heures de travail très élevé, pénibilité des tâches, retards ou non-paiement des salaires que vient renforcer le problème des garants fantômes. Lors de nos enquêtes, nombre de migrants et de responsables d'organisation nous ont en effet expliqué que les travailleurs migrants acceptent souvent des salaires très bas, ce qui se traduit par de longues journées de travail, parfois aussi par leur installation ou celle de leur famille sur le lieu du travail, par l'absence d'encadrement des enfants et même parfois par de longes

${ }^{1}$ Statistiques Disponible sur internet (consulté le 3 avril 2012). années d'endettement pour seulement rembourser le prix du voyage. Notons néanmoins que si les métiers de ces migrants au Liban sont généralement peu qualifiés et marqués par des conditions très difficiles sur les dix dernières années, le statut professionnel de certains migrants s'oriente toutefois vers des emplois plus gratifiants: par exemple, des femmes migrantes travaillent dans les boulangeries, les pâtisseries, les magasins, les salons de coiffure, les chocolateries et des hommes dans des ateliers de mécanique, des imprimeries, des pressings.

Le deuxième chapitre présente le rôle des ambassades et des associations caritatives dans la protection de la main d'œuvre étrangère et l'aide aux migrants. Aucune loi ne couvre les droits des travailleurs étrangers au Liban. Les ambassades, les organisations non gouvernementales (ONG) et les institutions religieuses sont les seules garantes des droits de ces migrants. Elles se déclinent en différentes structures d'accueil qui sont autant de lieux de convivialité pour ces travailleurs. L'implication des ambassades, du gouvernement et des ONG dans l'organisation et la gestion de l'immigration nous a conduit à nous interroger sur les politiques publiques relatives à ce phénomène tout en s'intéressant aux changements dans la vie de ces immigrés. Parmi ces acteurs, certains interviennent pour les défendre, ils prennent des mesures pour empêcher et réduire les abus à leur encontre et maintiennent de bonnes relations avec leurs employeurs. Enfin, nous nous sommes attachés à analyser la segmentation du marché du travail au Liban, selon les origines nationales et ethniques, mais aussi en fonction des contraintes auxquelles la société libanaise soumet les groupes et les individus.

\section{Dans la troisième partie de notre} thèse, nous avons présenté les grands traits qui caractérisent l'immigration 
contemporaine au Liban, en particulier les conditions de vie et les pratiques culturelles. Notre enquête a également permis de voir que de nouveaux arrivants étaient confrontés à des difficultés administratives, linguistiques, professionnelles et économiques. Au passage, notons qu'il a été difficile de rencontrer une partie des migrants, particulièrement des femmes. En effet, il n'est pas toujours facile pour ces dernières de sortir de la maison de leurs employeurs et elles ne peuvent donc ni participer aux fêtes, ni aux diverses activités de leur communauté. En comparant les données des quatre nationalités: les SriLankais, les Indiens, les Philippins et les Éthiopiens, nous avons décrit la vie communautaire de ces immigrés en nous basant sur un travail ethnographique et statistique. Nous avons pu ainsi observer que les pratiques culturelles de la maind'œuvre étrangère se traduisent par la préservation de coutumes et traditions à travers les cérémonies, les pratiques religieuses, le mariage et le concubinage, les liens avec le pays d'origine tels que le nombre de retours dans le pays d'origine, les contacts familiaux, les appels téléphoniques ou les courriers. Les résultats de notre enquête montrent aussi la cohabitation entre les migrants de différentes nationalités, notamment les Philippins et les Éthiopiens, ce qui est une caractéristique nouvelle au Liban. Du fait de l'augmentation des prix des chambres, ils habitent les banlieues de Beyrouth parce que les prix y sont plus attractifs. Les offres d'emplois y sont également plus nombreuses. Les informations recueillies dans cette thèse au sujet des conditions de vie des migrants témoignent d'actes racistes dans les sociétés arabes et libanaises soulignant un manque de considération pour ces immigrés. Nous avons aussi constaté des violences et des abus physiques et psychologiques de la part des employeurs envers les travailleurs migrants, notamment à l'intérieur des maisons.
D’une manière générale, notre recherche a confirmé l'idée que l'immigration au Liban devient un phénomène social ethnicisé. Nous avons analysé cette ethnicisation sous différents aspects : situation d'arrivée, emploi occupé, vie culturelle, retour vers le pays d'origine. Nous avons constaté qu'il existe des modes d'organisation spécifiques chez les migrants, avec une «structure sociale » parallèle à celle qui organise la société libanaise. Notre propos n'a pas été d'énumérer ni même de recenser les différences entre l'une et l'autre mais de tenter de voir comment elles fonctionnent l'une par rapport à l'autre et d'en repérer les caractéristiques principales. Nous avons constaté une ethnicisation sur le marché de l'emploi par la présence d'hommes et de femmes de différentes nationalités, en distinguant les migrants légaux et illégaux, et les différentes catégories socioprofessionnelles. De même, nous avons noté l'appropriation, par la population migrante, de nouveaux espaces, par ailleurs lieux collectifs, tels que les zones de commerces et les lieux de culte (mosquées, églises, etc.).

L'immigration au Liban est devenue une réalité sociale et économique qui alimente de plus en plus les débats sur l'intégration et la diversité, fait nouveau au Liban. Depuis quelques années, la question de l'immigration est présentée comme une des priorités des gouvernements successifs. D'un côté, le gouvernement libanais tente d'adopter une stratégie pour limiter le flux des étrangers et d'un autre, le Liban ne peut se passer de la main-d'œuvre étrangère dans de nombreux secteurs. Face à ce dilemme qui n'en est pas vraiment un, le Liban semble s'accommoder de ne pas avoir de véritable législation relative à l'entrée et au séjour des étrangers. Ainsi, les travailleurs étrangers sont confrontés à bien des difficultés pour faire respecter leurs droits, d'autant plus quand une part de la société et 
des autorités encouragent - par leur attitude « le travail au noir ».

Suzanne Menhem Enseignante Chercheure Université Libanaise Institut des Sciences Sociales-II

Rabieh-Liban MIGRINTER - UMR 7301 CNRS / Université de Poitiers suzanne.menhem@gmail.com
Sassen, Saskia (1999) La métropole: site stratégique et nouvelle frontière, Cultures \& Conflits, n³3-34, pp. 123-140.

\section{Bibliographie}

Courgeau, Daniel (1988) Méthodes de mesure de la mobilité spatiale: migrations internes, mobilité temporaire, navettes, Paris, éditions de l'INED, $302 \mathrm{p}$.

Domenach, Hervé ; Picouet, Michel (1987) Le caractère de réversibilité dans l'étude de la migration, Population, n³ 3 , pp. 469-484.

Dureau, Françoise (2002) Les systèmes résidentiels : concepts et applications, in Lévy, J.P. ; Dureau, F. (Éd.) L'accès à la ville. Les mobilités en question, Paris, L'Harmattan, pp. 355-382 (Habitat et sociétés).

Ma Mung, Emmanuel; Doraï, Mohamed Kamel ; Hily, Marie-Antoinette ; Loyer, Frantz (1998) Bilan des travaux sur la circulation migratoire. Rapport final, commandé par le Ministère de la Solidarité et de l'Emploi, Direction de la Population et des Migrations (DPM), Poitiers, Migrinter, 143 p. [Disponible sur Internet].

Menhem, Suzanne (2012) Les nowveaux migrants au Liban: vers une ethnicisation $d u$ marché de l'emploi, Poitiers, Université de Poitiers, 428 p. Th. doct: Sc. Sociales : Poitiers : Université de Poitiers, 428 p. 